\title{
Error propagation in the numerical integration of solitary waves. The regularized long wave equation
}

\author{
A. Araújo ${ }^{a}$, A. Durán ${ }^{b, *}$ \\ a Departamento de Matematica, Universidade de Coimbra, Apartado 3008, 3000 Coimbra, Portugal \\ b Departamento de Matemática Aplicada y Computación, Universidad de Valladolid, Paseo del Prado de la Magdalena s/n, \\ 47005 Valladolid, Spain
}

\begin{abstract}
We study the error propagation of time integrators of solitary wave solutions for the regularized long wave equation, $u_{t}+u_{x}+\frac{1}{2}\left(u^{2}\right)_{x}-u_{x x t}=0$, by using a geometric interpretation of these waves as relative equilibria. We show that the error growth is linear for schemes that preserve invariant quantities of the problem and quadratic for 'nonconservative' methods. Numerical experiments are presented. ๑ 2001 IMACS. Published by Elsevier Science B.V. All rights reserved.
\end{abstract}

Keywords: Hamiltonian structure; Solitary waves; Relative equilibria; Conservative methods; Symmetry groups

\section{Introduction}

The purpose of this paper is to analyze the growth with time of the error of time-integrators for solitary wave solutions of the regularized long wave (RLW) equation. For this problem, we establish a better error propagation for schemes that conserve invariants of the equation. These conservative methods exhibit a linear error growth, while for 'general' integrators, the propagation with time of the error is quadratic. The evolution of these invariants in the numerical integration is also influenced by the conservation properties of the scheme considered.

The advantages of geometric integrators [28], i.e., of methods that mimic geometric properties of the system of differential equations being integrated, are well known in the case of ordinary differential equations [5-7,14]. In Refs. [9,12], partial differential equations, as the Korteweg-de Vries equation and the nonlinear Schrödinger equation, have been analyzed. These works are in the origin of the present paper, along with the geometric study given in Ref. [11]. We prove that the mechanisms leading to favorable error propagation, that were studied to those equations, can be applied to this case.

\footnotetext{
* Corresponding author.

E-mail addresses: alma@mat.uc.pt (A. Araújo), angel@mac.cie.uva.es (A. Durán).
} 
Our results are based on several facts: first, the RLW equation admits a Hamiltonian structure [1]. It also possesses a symmetry group [1,23], generated by one of its invariants. In this context, solitary wave solutions arise from a relative equilibrium problem [1]; this means that these solutions are critical points of the Hamiltonian function restricted to level sets of the invariant associated to the symmetry group. This provides a geometric point of view to understand the role of the conserved quantities in the problem.

On the other hand, in order to approximate these traveling wave solutions, we need to investigate their stability. Several papers perform classical stability analyses for this kind of waves [2-4,34], but, for our numerical treatment, we make use of asymptotic stability results. The papers [26] and [33] were used in [9] and [12], respectively. In this case, asymptotic stability for RLW solitary waves were first observed numerically $[13,22]$ and the proof of this property is obtained in [21].

The benefits of conservative schemes for this problem are shown in some aspects. The first one, as we mentioned previously, is the more efficient behaviour in time of the error. Another point is that the numerical solution may admit an asymptotic expansion in which a new solitary wave appears. This is the so-called modified solitary wave, that comes from the original one through perturbations in time of the wave parameters: amplitude, velocity and initial location. We point out that these perturbations depend on the conservative character of the integrator considered. A final comment concerns the evolution of the conserved quantities through the numerical approximation. Here, the relative equilibrium problem, solved by the solitary waves, states a dependency between the invariants involved. This relation implies a better behaviour of the conservative methods for this matter.

The paper is structured as follows: Section 2 describes some relevant properties of the problem being considered, as the Hamiltonian structure of the equation and the symmetry group we will deal with; we also interpretate the solitary wave solutions as relative equilibria. In Section 3 we analyze the linearization of the equation around one of these solitary wave solutions, while Section 4 is devoted to the main results and to numerical experiments.

A natural extension of this paper concerns the application of these techniques to interactions of solitary waves. The equations studied in $[9,12]$ are examples of integrable partial differential equations: they possess an infinite number of conserved quantities and the so-called soliton property, for which the nonlinear interaction of solitary wave solutions leaves the waves basically unaltered. This is not the case of the RLW equation, which has only a finite number of invariants [24], which indicates that the equation is nonintegrable. The solitary wave interaction problem for the RLW equation has been studied numerically in several papers (see, for example, Refs. $[13,15,16,30]$ ) and, from this point of view, the present work can also be another starting-point in the treatment of this problem.

Some other generalizations of our analysis can be considered. Miller and Weinstein [21] extend their asymptotic stability analysis to the solitary wave solutions of the modified RLW equation, $u_{t}+u_{x}+\frac{1}{3}\left(u^{3}\right)_{x}-u_{x x t}=0$, and our numerical study could be applied here. On the other hand, the behaviour of higher order terms in the expansion of the error or different discretizations may also be the subject of future work. 


\section{The regularized long wave equation}

\subsection{Hamiltonian structure and symmetry groups}

The equation treated in the present case is the regularized long wave (RLW) equation

$$
u_{t}+u_{x}+\frac{1}{2}\left(u^{2}\right)_{x}-u_{x x t}=0, \quad-\infty<x<\infty, t>0 .
$$

Eq. (1) appears in many physical applications [8,27,35], and it has been studied extensively. A classical reference is Ref. [3]; in fact, (1) is also referred to as the Benjamin-Bona-Mahoney equation. Results on the existence of solutions can also be seen in Ref. [21] and numerical works concerning (1) are numerous as well $[8,13,15,16,30]$. In the present work, we focus on the Hamiltonian structure of (1). In this case, the phase space $\Omega$ for the Hamiltonian formalism consists of sufficiently smooth real functions $u$ of the spatial variable $x$ with its derivatives decreasing sufficiently fast at infinity. Thus, Eq. (1) can be expressed as the following infinite-dimensional evolution equation, in Hamiltonian form

$$
\frac{\mathrm{d} u}{\mathrm{~d} t}=\mathcal{J} \delta H(u)
$$

where $\mathcal{J}$ is the skew-adjoint operator [3,21,27],

$$
\mathcal{J}=-\left(1-\partial_{x x}\right)^{-1} \partial_{x}
$$

$\delta$ denotes variational derivative and the energy $H$ is given by

$$
H(u)=\int_{-\infty}^{\infty}\left(\frac{1}{2} u^{2}+\frac{1}{6} u^{3}\right) \mathrm{d} x .
$$

The Hamiltonian structure associated to the operator (3) is determined by the Poisson bracket [20,23]

$$
\{F, G\}=\int_{-\infty}^{\infty} \delta F \mathcal{J} \delta G \mathrm{~d} x .
$$

Observe that (5) admits Casimir functions [20,23]; they are functionals with zero Poisson bracket with any other functional. In particular, they give rise to trivial conserved quantities of Eq. (1), see Ref. [23]. In the subsequent analysis we make use of one of these Casimir functions

$$
C(u)=\int_{-\infty}^{\infty} u \mathrm{~d} x,
$$

which we call 'mass'. The RLW equation also admits another conserved quantity ('momentum')

$$
I(u)=\int_{-\infty}^{\infty}\left(\frac{1}{2} u^{2}+\frac{1}{2} u_{x}^{2}\right) \mathrm{d} x .
$$

Observe that $\delta I(u)=1-\partial_{x x} u$; then the Hamiltonian vector field $v_{I}=\mathcal{J} \delta I(u)=-u_{x}$ associated with $I$, is the infinitesimal generator [23] of the one-parameter symmetry group of Eq. (1),

$$
G_{\varepsilon}(u(x))=u(x-\varepsilon), \quad \varepsilon \in \mathbb{R},
$$


that consists of translations in the spatial variable. This means that $G_{\varepsilon}$ is the flow of the vector field $v_{I}$. In terms of the Poisson bracket (5), the condition for $H$ to admit the symmetry group (8) is $\{I, H\}=$ constant. In fact, since $I$ is a first integral of (1), we have $\{I, H\}=0$. The Casimir function (6) is not associated to any other group.

\subsection{Solitary wave solutions}

Eq. (1) admits a two-parameter family of solitary wave solutions [10,35] that can be seen as relative equilibria [1] associated to the symmetry group (8). The reduction described in Refs. [11,12] can be applied to this case. Here, the phase space $\Omega$ is foliated by level sets $\{I=k\}$ of the first integral $I$. These level sets are manifolds that, because of the involution condition $\{I, H\}=0$, are invariant by the flow of (1), that is an initial condition on $\{I=k\}$ leads to a solution of (1) remaining on this level set for all t. On the other hand, due to the fact that $\{I, I\}=0$, each orbit $\left\{G_{\varepsilon}(u), \varepsilon \in \mathbb{R}\right\}$ of the symmetry group is contained in a level set $\{I=k\}$. Thus, each of these level sets is foliated by orbits of the group and we can construct the corresponding reduced phase space [1,23], that is, the quotient space consisting of points that are orbits in the original phase space, determined by the value $k$. On each reduced phase space, the original Hamiltonian system leads to a new Hamiltonian system, the reduced system [1,23], that governs the time evolution of the group orbits, that is the evolution of $u$ modulo translations in $x$.

For a fixed level set $\{I=k\}$ we look for relative equilibria $u_{0} \in \Omega$ [1]

$$
\begin{aligned}
& \delta H\left(u_{0}\right)-c \delta I\left(u_{0}\right)=0, \\
& I\left(u_{0}\right)=k,
\end{aligned}
$$

for, initially, $c \in \mathbb{R}$. We are looking for stationary points of $H$ restricted to the level set. The group orbit through a relative equilibrium $u_{0}$ is an equilibrium of the reduced system. The condition (9) for $u_{0}$ generates a solution of (1) from this initial value in a simple way, given by the one-parameter symmetry subgroup: $u(t)=G_{t c}\left(u_{0}\right)$ [11]. Then, the time evolution of the initial profile is given by a translation governed by parameters growing linearly with time.

The substitution of (9) for this case gives

$$
c u_{0}^{\prime \prime}-(c-1) u_{0}+\frac{1}{2} u_{0}^{2}=0,
$$

generating, for $c>1$, the solution

$$
u_{0}(x)=3(c-1) \operatorname{sech}^{2}\left(\frac{1}{2} \sqrt{1-\frac{1}{c} x}\right) \text {. }
$$

The level set (10) determines the relation with the parameter $c$ :

$$
k=\frac{12(c-1)^{2}}{\sqrt{1-1 / c}}\left(1+\frac{1}{5}\left(1-\frac{1}{c}\right)\right) .
$$

The group orbit through $u_{0}$ gives rise to a one-parameter family of solutions

$$
\varphi\left(x, x_{0}\right)=G_{x_{0}}\left(u_{0}\right)=u_{0}\left(x-x_{0}\right) .
$$

Every solution (12) projects onto the same equilibrium in the reduced phase space. Finally, we obtain the solutions of (1) given by

$$
\psi\left(x, t, c, x_{0}\right)=G_{t c}(\varphi)=3(c-1) \operatorname{sech}^{2}\left(\frac{1}{2} \sqrt{1-\frac{1}{c}}\left(x-c t-x_{0}\right)\right) .
$$


This is a two-parameter family of solitary wave solutions. The parameter $c$ determines not only the velocity of the wave but also its amplitude (compare with Refs. [9,12]), with the taller the wave the faster it travels. On the other hand, $x_{0}$ governs the initial location.

\section{The variational equation}

\subsection{The homogeneous linearized equation}

The time propagation of the error in the numerical integration of solitary wave problems for (1) can be studied in terms of the corresponding linearized equation of (1) near a relative equilibrium solution like $\psi$ in (13). If $\delta$ denotes the perturbation of $\psi$ from an initial small change $\psi\left(x, 0, c, x_{0}\right)+\varepsilon \delta_{0}(x)$, then $\delta$ satisfies the homogeneous variational equation

$$
\left(1-\partial_{x x}\right) \delta_{t}+\partial_{x}(\delta+\psi \delta)=0 .
$$

With the change of variables given by the symmetry group $\delta=G_{t c \Delta}$ [12] we can write (14) as

$$
\Delta_{T}-\Delta_{\xi \xi T}+c \Delta_{\xi \xi \xi}+\partial_{\xi}((1-c) \Delta+\psi \Delta)=0,
$$

with $\xi=x-c t-x_{0}, T=t$. Now, (15) can be expressed as an evolution equation

$$
\Delta_{T}=L \Delta \text {, }
$$

with the operator $L$ given by [21]

$$
\begin{aligned}
& L=\left(1-\partial_{\xi \xi}\right)^{-1} \partial_{\xi} L_{c}, \\
& L_{c}=-c \partial_{\xi \xi}+c-1-\psi(\xi) .
\end{aligned}
$$

As in Ref. [12] we will also treat with the corresponding nonhomogeneous variational equation for source terms that admit $\left\{G_{t c}: t \in \mathbb{R}\right\}$ as a symmetry group. By using the change of variables above, we can present this nonhomogeneous case as

$$
\Delta_{T}=L \Delta+s,
$$

with $s$ constant.

In order to study (16) in more detail, we consider the Sobolev space $H_{a}^{1}[21,26]$ that consists of functions $v(\xi)$ such that $\mathrm{e}^{a \xi} v(\xi) \in H^{1}(\mathbb{R})$, for a constant $a$ with $0<a<\sqrt{1-1 / c}$. The norm considered is

$$
\|v\|_{H_{a}^{1}}=\left\|\mathrm{e}^{a \xi} v\right\|_{H^{1}} .
$$

Observe that the solitary wave $\psi(\xi)$ and its derivatives belong to this space.

The spectral properties of the operator $L$ are analyzed in Ref. [21]:

Lemma 3.1. There exists $c^{*}>1$ such that for all $c \in\left(1, c^{*}\right]$ and for all but a discrete set of $c \geqslant c^{*}, \lambda=0$ is the only eigenvalue of $L$ in $H_{a}^{1}$ with $\operatorname{Re} \lambda \geqslant 0$, where $0<a<\sqrt{1-1 / c}$. The geometric multiplicity of this eigenvalue is 1 and the algebraic multiplicity is 2 . The generalized kernel of $L, \operatorname{Ker}_{g} L$, is spanned by the functions 


$$
\begin{aligned}
& \Phi_{1}(\xi)=\frac{\partial \psi}{\partial x_{0}}, \\
& \Phi_{2}(\xi)=\frac{\partial \psi}{\partial c},
\end{aligned}
$$

with $L \Phi_{1}=0, L \Phi_{2}=\Phi_{1}$.

Then, (16) has solutions of the form

$$
\alpha \Phi_{1}=\beta\left(\Phi_{2}+T \Phi_{1}\right),
$$

which, in terms of the original variables $x, t$, are linear combinations of the partial derivatives of the solitary wave with respect to $x_{0}$ and $c$. They represent changes of the wave due to the perturbation of its parameters. Explicitly, $\Phi_{1}$ corresponds to the group generator $v_{I}$ at the solitary wave and therefore induces changes in the location $x_{0}$. On the other hand, $\Phi_{2}$ is associated to modifications in the solitary wave corresponding to changes in the value of $I$, determined by $c$.

We denote by $P$ the projection of $H_{a}^{1}$ onto the generalized kernel of $L$ and by $Q$ its complement $I-P$, that is,

$$
\begin{aligned}
P_{v} & =\left\langle v, \Psi_{1}\right\rangle \Phi_{1}+\left\langle v, \Psi_{2}\right\rangle \Phi_{2}, \\
Q_{v} & =v-P v .
\end{aligned}
$$

Here, $\Psi_{1}$ and $\Psi_{2}$ form a basis of the generalized kernel of the adjoint operator $L^{*}$ of $L$, where $\langle\cdot, \cdot\rangle$ denotes the $L^{2}$ inner product. These functions can be chosen in such a way that $\left\langle\Phi_{i}, \Psi_{j}\right\rangle=\delta_{i j}$. More precisely [21],

$$
\begin{aligned}
& \Psi_{1}=\theta_{1}\left(\partial_{\xi} \Phi_{2}-\int_{-\infty}^{\xi} \Phi_{2}(y) \mathrm{d} y\right)+\theta_{2}\left(1-\partial_{\xi \xi}\right) \psi, \\
& \Psi_{2}=\theta_{3}\left(1-\partial_{\xi \xi}\right) \psi,
\end{aligned}
$$

where

$$
\theta_{1}=-\frac{1}{(\mathrm{~d} / \mathrm{d} c) I(\psi)}, \quad \theta_{2}=-\frac{1}{2}\left(\frac{(\mathrm{d} / \mathrm{d} c) C(\psi)}{(\mathrm{d} / \mathrm{d} c) I(\psi)}\right)^{2}, \quad \theta_{3}=\theta_{1} .
$$

In fact, $L^{*} \Psi_{2}=0$ and $L^{*} \Psi_{1}=-\Psi_{2}$. Observe that $\Psi_{2}$ is essentially the gradient of the invariant $I$ evaluated at the solitary wave $\psi$.

An important result to analyze the solutions of the nonhomogeneous version (19) is also due to Miller and Weinstein [21]. They show that the operator $L$ generates a $C_{0}$ semigroup with exponentially decaying $H_{a}^{1}$ norm on the spectral complement of its generalized kernel.

Lemma 3.2. Assume that $0<a<\sqrt{1-1 / c}$ and that $\lambda=0$ is the only eigenvalue of $L$ in $H_{a}^{1}$ with $\operatorname{Re} \lambda \geqslant 0$. Let $Q$ denote the projection onto $\operatorname{Ker}_{g}\left(L^{*}\right)^{\perp}$ (see (22)). If $\Delta(T)$ is a solution of (16) with initial condition $\Delta_{0} \in H_{a}^{1} \cap Q\left(H_{a}^{1}\right)$, then $\Delta(T) \in H_{a}^{1} \cap Q\left(H_{a}^{1}\right)$ for all $T$ and there are constants $k_{1}, k_{2}>0$ such that

$$
\|\Delta(T)\|_{H_{a}^{1}} \leqslant k_{1} \mathrm{e}^{-k_{2} T}\left\|\Delta_{0}\right\|_{H_{a}^{1}} .
$$


That is, solutions of (16) with initial data in $X=H_{a}^{1} \cap \operatorname{Ker}_{g}\left(L^{*}\right)^{\perp}$ decay exponentially to zero in $H_{a}^{1}$ norm as $t \rightarrow \infty$. As far as the $H^{1}$ norm is concerned, observe that solutions of (16) conserve the functional

$$
F(\Delta)=\int_{-\infty}^{\infty}\left(\frac{c}{2}\left(\partial_{\xi} \Delta\right)^{2}+\frac{(c-1)}{2} \Delta^{2}-\frac{1}{2} \psi \Delta^{2}\right) \mathrm{d} \xi,
$$

(recall that $c>1$ ); therefore, if $\Delta(T)$ is a solution of (16), we have

$$
\begin{aligned}
\|\Delta(T)\|_{H^{1}}^{2} & \leqslant \frac{2}{c-1} F(\Delta(T))+\frac{1}{c-1} \int_{-\infty}^{\infty} \psi \Delta^{2} \mathrm{~d} \xi \\
& =\frac{2}{c-1} F\left(\Delta_{0}\right)+\frac{1}{c-1} \int_{-\infty}^{\infty} \psi \mathrm{e}^{-2 a \xi} \Delta^{2} \mathrm{e}^{2 a \xi} \mathrm{d} \xi \\
& \leqslant \frac{2}{c-1} F\left(\Delta_{0}\right)+C\|\Delta(T)\|_{H_{a}^{1}}\|\Delta(T)\|_{H^{1}} \\
& \leqslant \frac{2}{c-1} F\left(\Delta_{0}\right)+\frac{1}{2}\|\Delta(T)\|_{H^{1}}^{2}+C^{\prime}\|\Delta(T)\|_{H_{a}^{1}}^{2} .
\end{aligned}
$$

Now, $F\left(\Delta_{0}\right)$ can be written in terms of $\left\|\Delta_{0}\right\|_{H^{1}}$ and we have the following estimate:

Lemma 3.3. Under the conditions of Lemma 3.2, if $\Delta_{0} \in X \cap H^{1}$, then there exists $k_{1}, k_{2}>0$ such that

$$
\|\Delta(T)\|_{H^{1}} \leqslant k_{1}\left(\left\|\Delta_{0}\right\|_{H^{1}}+\mathrm{e}^{-k_{2} T}\left\|\Delta_{0}\right\|_{H_{a}^{1}}\right) \text {. }
$$

Observe that, since the symmetry group (8) consists of translations, the growth with time of solutions of (14) is similar to that of solutions of (16). The estimate (25) implies that the only source of growth with time of these solutions comes from its component in the generalized kernel of $L$, that is the term that represents perturbations in the parameters of the solitary wave.

\subsection{The nonhomogeneous variational equation}

The results above are essential to study the solutions of (18). If $s=s(\xi) \in H_{a}^{1}$, we can decompose $s=s_{P}+s_{Q}$ with $s_{P} \in \operatorname{Ker}_{g} L, s_{Q}=Q s \in X$. By using Lemma 3.1, we can write $s_{P}=s_{1}+s_{2}$ with $s_{1} \in \operatorname{Ker} L$ and $s_{2}$ in a supplement of $\operatorname{Ker} L$ in $\operatorname{Ker}_{g} L$. More precisely,

$$
s_{1}=\alpha_{1} \Phi_{1}, \quad s_{2}=\alpha_{2} \Phi_{2},
$$

with

$$
\alpha_{i}=\left\langle s, \Psi_{i}\right\rangle, \quad i=1,2 .
$$

In this context, we have

Lemma 3.4. If $0<a<\sqrt{1-1 / c}$ and $s \in H_{a}^{1}$, the solution of (19) with zero initial condition can be written in the following way:

$$
\Delta(T)=\alpha_{1} T \Phi_{1}+\alpha_{2}\left(T \Phi_{2}+\frac{T^{2}}{2} \Phi_{1}\right)+\Gamma(T),
$$


where $\alpha_{1}, \alpha_{2}$ are given by (26). The function $\Gamma$ is of the form

$$
\Gamma(T)=\int_{0}^{T} \exp ((T-\tau) L) s_{Q} \mathrm{~d} \tau .
$$

Moreover, if $s_{Q} \in H^{1}$ and there exists $\beta \in H^{1} \cap H_{a}^{1}$ such that $s_{Q}=L \beta$, then $\Gamma(T)$ remains, for all $T$, bounded in the $H^{1}$ norm.

Proof. The expression (27) can be proved by using Duhamel's principle and the previous results in a similar way to that of Ref. [12]. Observe that we can write

$$
\Delta(T)=T s_{1}+\left(T I+\frac{T^{2}}{2} L\right) s_{2}+\int_{0}^{T} \exp ((T-\tau) L) s_{Q} \mathrm{~d} \tau,
$$

where $I$ is the identity operator. The substitution of $s_{1}$ and $s_{2}$ proves the first part of the lemma. Note that the estimate (25) shows that, if $s_{Q} \in H^{1}$, then $\Gamma(T)$, with a bounded integrand, grows at most linearly with time in the $H^{1}$ norm. On the other hand, if $Q_{s}$ is in the range of the operator $L, Q_{s}=L \beta$ for $\beta \in H^{1} \cap X$ (note that $Q\left(H_{a}^{1}\right)$ is invariant by $L$ ), then we can write $\Gamma(T)$ in the form

$$
\Gamma(T)=(\exp (T L)-I) \beta,
$$

and we use Lemma 3.3 to conclude the proof.

\section{Remarks.}

(a) Note that the first two terms in (27) correspond to the projection of the solution on $\mathrm{Ker}_{g} L$, with its components in $\operatorname{Ker} L\left(T s_{1}\right.$ and $\left.\left(T^{2} / 2\right) L s_{2}\right)$ lying in the direction of the group generator at the solitary wave solution. The component in $\operatorname{Ker}_{g} L$ but not in $\operatorname{Ker} L$ corresponds to the variation of the relative equilibrium with respect to the parameter $c$, that governs the level manifold. Then, this first part represents perturbations in the wave parameters, growing quadratically with time in the direction of $\operatorname{Ker} L$ (in the group parameter $x_{0}$ ) and linearly in the level set parameter $c$. If the source term $s$ is orthogonal to the gradient of $I$ evaluated at the solitary wave, then $s_{2}=0$ and the growth is linear.

(b) The behaviour of the integral $\Gamma(T)$ in (28) shows that the perturbations that do not correspond to changes in wave parameters grow at most linearly with time in the $H^{1}$ norm. In the following section, we deal with smooth source terms $s=s(\xi)$ in $H^{1} \cap H_{a}^{1}$ which are orthogonal to the gradients of the invariants $C$ and $I$ at the solitary wave. In this case, these perturbations can be controlled in $H^{1}$ norm uniformly in time. To see this, we first observe that the orthogonality conditions imply

$$
\langle s, 1\rangle=\left\langle s, \Psi_{2}\right\rangle=0,
$$

with $\Psi_{2}$ given by (23). Therefore,

$$
\langle Q s, 1\rangle=\langle s, 1\rangle-\left\langle s, \Psi_{1}\right\rangle\left\langle\Phi_{1}, 1\right\rangle-\left\langle s, \Psi_{2}\right\rangle\left\langle\Phi_{2}, 1\right\rangle=\left\langle s, \Psi_{1}\right\rangle\left\langle\Phi_{1}, 1\right\rangle=0
$$

(the last expression vanishes because $\left\langle\Phi_{1}, 1\right\rangle=0$ ). Then we can define

$$
F(\xi)=\int_{-\infty}^{\xi} Q s(y) \mathrm{d} y
$$


that is a smooth function in $H^{1} \cap H_{a}^{1}$ such that $\partial_{\xi} F=Q s$. On the other hand, the operator $L$ can be written as

$$
L=\partial_{\xi} M_{c}, \quad M_{c}=\left(1-\partial_{\xi \xi}\right)^{-1} L_{c},
$$

and $L_{c}$, given by (18), is selfadjoint in $L^{2}$ with $\operatorname{Ker} L_{c}=\operatorname{span}\left(\partial_{\xi} \psi\right)$ [33]. Now, we have

$$
\left\langle\left(1-\partial_{\xi \xi}\right) F, \partial_{\xi} \psi\right\rangle=-\left\langle\partial_{\xi} F,\left(1-\partial_{\xi \xi}\right) \psi\right\rangle=-\frac{1}{\theta_{2}}\left\langle Q s, \Psi_{2}\right\rangle=0 .
$$

This means that $F$ is in the range of $M_{c}$; therefore, $Q s$ is in the range of $L$ and the last part of Lemma 3.4 applies.

\section{Numerical approximation}

\subsection{Main results}

We make use of the preceding theoretical results in order to study the behaviour in time of approximations to a solitary wave of the family (13). We consider a semidiscrete (discrete $t$, continuous $x$ ) one-step integrators for the initial value problem for (1) of the form

$$
U^{n+1}=\chi_{\Delta t}\left(U^{n}\right)
$$

where $\Delta t$ denotes the time step, $U^{n}=U^{n}(x)$ is a numerical solution at time level $t_{n}=n \Delta t, n=0,1, \ldots$, and $\chi_{\Delta t}$ approximates the flow of the equation. Thus, if $U^{0}=u_{0}$, then $U^{n}$ is an approximation to the value $u\left(t_{n}\right)$ of the solution $u$ of (1) with initial condition $u_{0}$.

We make some additional hypotheses about the method (29). Taking $u_{0}$ as the relative equilibrium $\varphi$ like (12) and if the corresponding solution $\psi$ in (13) is approximated by (29) with $U^{0}=\varphi$, we first assume that the local error at $\psi$, that is the difference between the true $\Delta t$-flow at $\psi$ and $\chi_{\Delta t}(\psi)$, admits an expansion of the form

$$
\Delta t^{r+1} l(\psi)+\Delta t^{r+1} R(\psi, \Delta t)
$$

where $r$ is the order of the method, $l, R$ are mappings defined in $\Omega$ with values in $\Omega, l$ is independent of $\Delta t$ and $\|R(\cdot, \Delta t)\|_{H^{1}} \rightarrow 0$ as $\Delta t \rightarrow 0$. It is also reasonable to suppose that $l=l(\psi)$ is a smooth function in $H^{1} \cap H_{a}^{1}$, since this mapping depends on $\psi$ and its derivatives, which belong to this space [9].

The second hypothesis states the invariance of the mapping $\chi_{\Delta t}$ by the one-parameter group $\left\{G_{t c}\right.$ : $t \in \mathbb{R}\}$ so that the mapping $l$ in (30) admits this group as a symmetry group. Note that, since the group consists of translations and most standard integrators are invariant with respect to linear transformations [31], this condition is not restrictive.

Finally, we also assume that the global error $U^{n}(x)-\psi\left(x, t_{n}\right)$ has an asymptotic expansion of the form

$$
U^{n}(x)-\psi\left(x, t_{n}\right)=\Delta t^{r} e\left(x, t_{n}\right)+\Delta t^{r} q\left(x, t_{n}, \Delta t\right), \quad-\infty<x<\infty,
$$

where the function $e$ is independent of $\Delta t$ and satisfies the corresponding nonhomogeneous variational equation [7]

$$
\begin{aligned}
& \left(1-\partial_{\xi \xi}\right) e_{t}+\partial_{x}(e+\psi e)=-l(\psi), \quad-\infty<x<\infty, t>0, \\
& e(x, 0)=0 .
\end{aligned}
$$


Moreover, $q$ is a remainder that, for fixed $t$, tends to zero in the $H^{1}$ norm as $\Delta t \rightarrow 0$.

The following theorem describes the asymptotic behaviour with time of the approximation to the solitary wave, given by (29).

Theorem 4.1 (i) (General case). Assume that (30), (31) hold and $\chi_{\Delta t}$ is invariant by the one-parameter group $\left\{G_{t c}: t \in \mathbb{R}\right\}$. Then, we have

$$
U^{n}(x)=\psi\left(x, t_{n}, \tilde{c}, \tilde{x}\right)+\Delta t^{r} \rho\left(x, t_{n}\right)+\Delta t^{r} Q\left(x, t_{n}, \Delta t\right),
$$

where

$$
\begin{aligned}
& \tilde{c}=c+\alpha_{2} t_{n} \Delta t^{r}, \\
& \tilde{x}=x_{0}+\left(\alpha_{1} t_{n}+\alpha_{2} \frac{t_{n}^{2}}{2}\right) \Delta t^{r},
\end{aligned}
$$

with $\alpha_{i}=-\left\langle l, \Psi_{i}\right\rangle, i=1,2$. The function $\rho$ is independent of $\Delta t$ and there is a constant $C>0$ such that $\|\rho(\cdot, t)\|_{H^{1}} \leqslant C t$. The function $Q$ is a remainder such that, for fixed $t,\|Q(\cdot, t, \Delta t)\|_{H^{1}} \rightarrow 0$ as $\Delta t \rightarrow 0$.

(ii) (Conservative case). If the method (29) satisfies

$$
\langle l, \delta I(\varphi)\rangle=0,
$$

then (33)-(34) hold with $\alpha_{2}=0$. Moreover, if

$$
\langle l, 1\rangle=0,
$$

then the function $\rho$ is bounded in the $H^{1}$ norm uniformly in time.

Proof. The proof of (i) is similar to that of Ref. [12]. Observe that the term $\rho$ corresponds to the component of the function $e$ that lies in the spectral complement of the generalized kernel of $L$ [27]. By using Lemmas 3.3 and 3.4 this term grows, in general, at most linearly in time. On the other hand, if the method satisfies (35), since $\Psi_{2}$ is, except multiplicative constant, the gradient of the invariant at the solitary wave (see (23)), then it is clear that $\alpha_{2}=0$.

It remains to prove the last part of (ii). But if (35), (36) hold, then Remark (b) of Section 3.2 applies and the function $\rho$ is bounded in the $H^{1}$ norm.

From the expansion (32) we note that the so-called modified solitary wave $[9,12]$, that is the first term of the right hand side of (32), has a new velocity $\tilde{c}$ given by (33) (and, therefore, a new amplitude) that is a linear with time perturbation of the velocity $c$ of the original wave. The new location $\tilde{x}$, given by (34), differs from the original $x_{0}$ in terms that grow quadratically with time. If the method conserves the quantity $I$, then [9] it satisfies (35) and the modified wave keeps the original velocity (and, therefore, amplitude) while the perturbation of $x_{0}$, that determines the modified location $\tilde{x}$, reduces its growth, being only linear. Note that the same conclusion can be reached if the method preserves the Hamiltonian (4) instead of $I$. In this case, the leading term of the local error is orthogonal to the gradient of $H$ at the solitary wave; but the relative equilibrium condition (9) implies that this gradient is proportional to the gradient of $I$ at this wave and, therefore, the scheme also satisfies (35).

We can also note that, if the method conserves the Casimir function $C$, then (36) holds. But, since $C$ is a linear functional, it is preserved by practically all methods used in practice [17], so (36) is not an exigent condition. 
The second term $\Delta t^{r} \rho$ of the right hand side of (32) is a complementary term [9,12] that represents errors of leading order $\mathrm{O}\left(\Delta t^{r}\right)$ not associated to changes in the parameters of the wave. Recall that it corresponds to the component of the leading term of the global error in $X$. In the 'conservative' case, this term is bounded in the $H^{1}$ norm, being necessary to impose the conservation not only of the quantity $I$ but also of the Casimir $C$ in order to obtain this bounded behaviour. Note that the invariant $C$ does not seem to play any role in the perturbation of the parameters of the solitary wave solution (see (33), (34)) while we can see the influence of the invariant $I$ in both the modified wave and the complementary term.

Finally, the third term in (32) $\Delta t^{r} Q$ is a remainder of higher order $\mathrm{o}\left(\Delta t^{r}\right)$.

\subsection{Numerical experiments}

The purpose of the experiments below is to show some advantages in the use of numerical schemes that conserve some invariant in the numerical integration of the solitary wave problem for (1), rather than to compare the efficiency of the methods. This idea will influence not only in the type of experiments being presented, but also in the choice of the integrators being used.

- From this point of view, we first consider the well-known implicit midpoint rule, which we call $[\mathrm{ICM}]$. This method has order two and conserves quadratic invariants of the system being integrated [29]. In our case, it preserves the quantity $I$ but not the Hamiltonian (4).

- We can interchange the invariant to be preserved and construct a second-order method [HCM] that conserves the Hamiltonian but not the functional $I[19,32]$. This scheme can be written as

$$
\begin{aligned}
& U^{n+1}=U^{n}-\Delta t\left(1-\partial_{x x}\right)^{-1} \partial_{x}\left(U^{n+1 / 2}+G\left(U^{n}, U^{n+1}\right)\right), \\
& U^{n+1 / 2}=\frac{U^{n}+U^{n+1}}{2}, \\
& G\left(U^{n}, U^{n+1}\right)=\frac{F\left(U^{n+1}\right)-F\left(U^{n}\right)}{U^{n+1}-U^{n}}, \quad F(U)=\frac{1}{6} U^{3} .
\end{aligned}
$$

- Finally, the Simply Diagonally Implicit Runge-Kutta method [NCM],

$$
\begin{array}{rr}
\gamma & 0 \\
1-2 \gamma & \gamma \\
\hline \frac{1}{2} & \frac{1}{2}
\end{array}
$$

with $\gamma=(3+\sqrt{3}) / 6$ and order three (see Ref. [18]), has been chosen to illustrate the behaviour of a typical nonconservative scheme, because this method does not preserve any of the quantities $I, H$.

It is not difficult to prove that the three methods considered conserve the Casimir function $C$. In fact, we have preferred to use schemes with some degree of competitivity instead of to construct mass nonpreserving integrators.

We present results concerning approximations to the solitary wave (13) with parameters $c=2$ (amplitude 3) and $x_{0}=-10$. To implement the methods, we use a fully aliased pseudospectral spatial discretization in such a way that, virtually, errors obtained correspond only to the time discretization [9]. This makes possible to observe the error growth with time in more detail. We successively refine the spatial grid until one is found for which no further error reduction is possible. On the other hand, 
standard analysis can check the validity of the hypotheses of the Theorem 4.1 for the three integrators considered [12]. We classify the numerical experiments presented in three classes:

- Differences in long time behaviour of the approximations in connection with conservation properties.

- Differences between the elements of the solitary wave solution and the form of the corresponding numerical approximation.

- Differences in time behaviour of the invariants $I$ and $H$ through numerical integration.

We first study the propagation in time of the global error of each method. Fig. 1 shows the $L^{2}$ norm of the error as a function of time, in log-log scale, up to $t_{\max }=200$. Here, the solid lines correspond to $[\mathrm{ICM}]$ and the dashed lines to [NCM]. Fig. 2 only differs from Fig. 1 in the scheme associated to the solid lines, that now represent [HCM]. The step sizes (for the two figures) are $\Delta t=\frac{1}{20}, \frac{1}{40}, \frac{1}{80}, \frac{1}{160}$.

The results of Table 1, which gives the errors at the final time $t_{\max }=200$ for the three methods, show the order of convergence, $r=2$ for [ICM] and [HCM] and $r=3$ for [NCM]. This can also be observed in Figs. 1 and 2 by watching the distance between parallel lines corresponding to a given method. On the other hand, the slopes of the lines show that, for [ICM] and [HCM], errors grow linearly with time, while for $[\mathrm{NCM}]$ the growth is quadratic after $t=10$ (compare with the lines plotted in the right down corner of the figures). Recall that the second-order methods [ICM] and [HCM] conserve, respectively, the momentum $I$ and the Hamiltonian $H$; according to Theorem 4.1, the parameters of the solitary wave solution are perturbed in terms that grow at most linearly with time. The behaviour in error propagation must be different in the case of [NCM], that does not preserve any of the quantities and, in fact, the leading term of its local error does not satisfy (35); here, the perturbations of the parameters grow quadratically with time.

From Figs. 1 and 2 we can also observe that, for some values of the time step $\Delta t$, [ICM] and [HCM] give smaller errors at the final time $t_{\max }=200$ than the third order scheme [NCM] (see, for example, the lines corresponding to $\Delta t=\frac{1}{20}, \frac{1}{40}$ ). The different behaviour with time of the error propagation suggests that, for long time integrations, it seems to be advisable to use an integrator with some conservation property.

The conservative character of a scheme has influence not only on the perturbations of the parameters of the wave (and therefore in the construction of the corresponding modified solitary wave) but also on the behaviour in time of the complementary term (see Theorem 4.1). This can be analyzed numerically, for moderate values of $t$, by computing the parameters of the modified solitary wave $\tilde{c}$ and $\tilde{x}$, and by measuring the errors between this new wave and the corresponding numerical solution. Table 2 gives these modified errors, in $L^{2}$ norm, for the three schemes, at the final time $t_{\max }=200$. Observe that in all cases, the modified error for any of the values of $\Delta t$ considered is smaller than the corresponding true error (compare with Table 1). Most of the error is incorporated to the perturbations of the parameters that determined the modified solitary wave. Figs. 3 and 4 illustrate the evolution in time of the modified error for $[\mathrm{HCM}]$ and $[\mathrm{NCM}]$, respectively (the case of $[\mathrm{ICM}]$ is similar to that of $[\mathrm{HCM}]$ and it provides no further information). Recall that the theoretical results reveal that, in the conservative case, the complementary term is bounded in time, while in the general case, the growth of this element is at most linear with $t$. This different behaviour is shown in Fig. 3, reflecting the bounded behaviour in the conservative case, and in Fig. 4, where error grows linearly with time.

Note that, since we only analyze the evolution in time of the leading term of the global error, not many things can be said about the remainder, whose behaviour in time should also affect in some way. At this point, two facts can be remarked. First, from Table 2 we see that, in the case of the second-order methods 


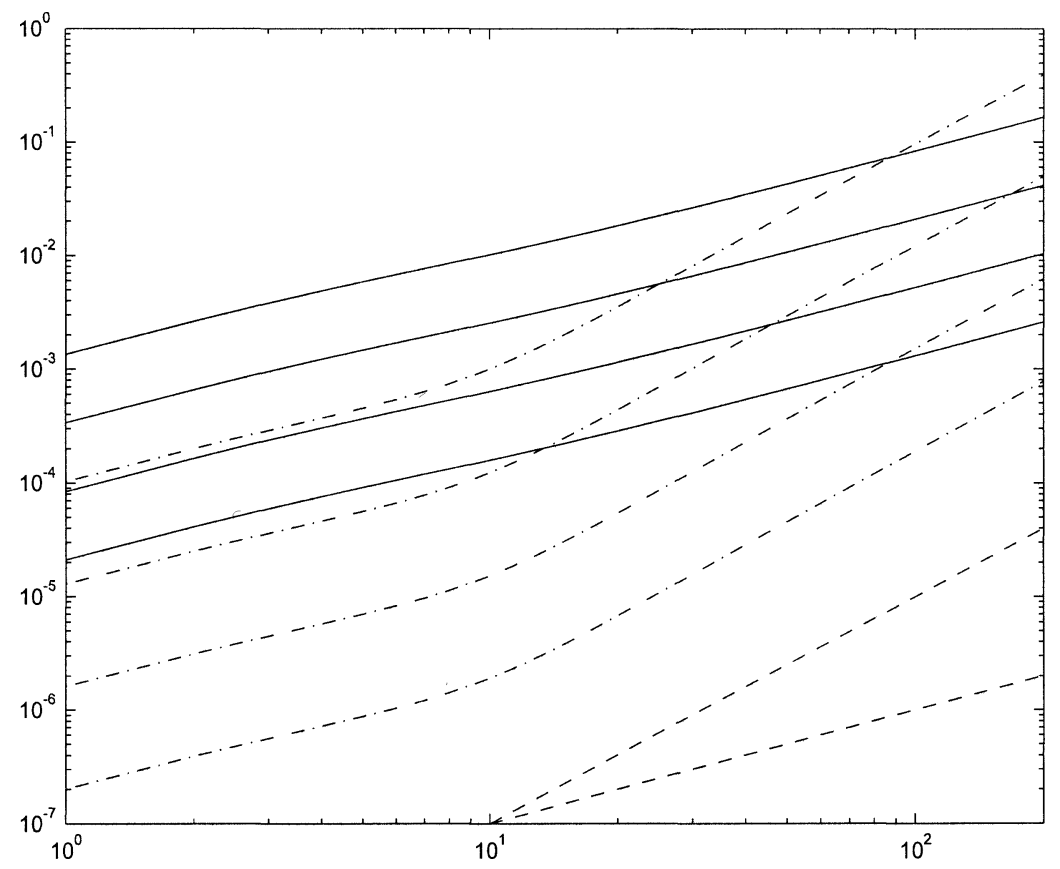

Fig. 1. $L^{2}$-error against $t$. Solid line: [ICM]; dashed line: [NCM]. The time steps are $\Delta t=\frac{1}{20}, \frac{1}{40}, \frac{1}{80}, \frac{1}{160}$. The broken lines at the bottom show the slopes for linear and quadratic growth in time.

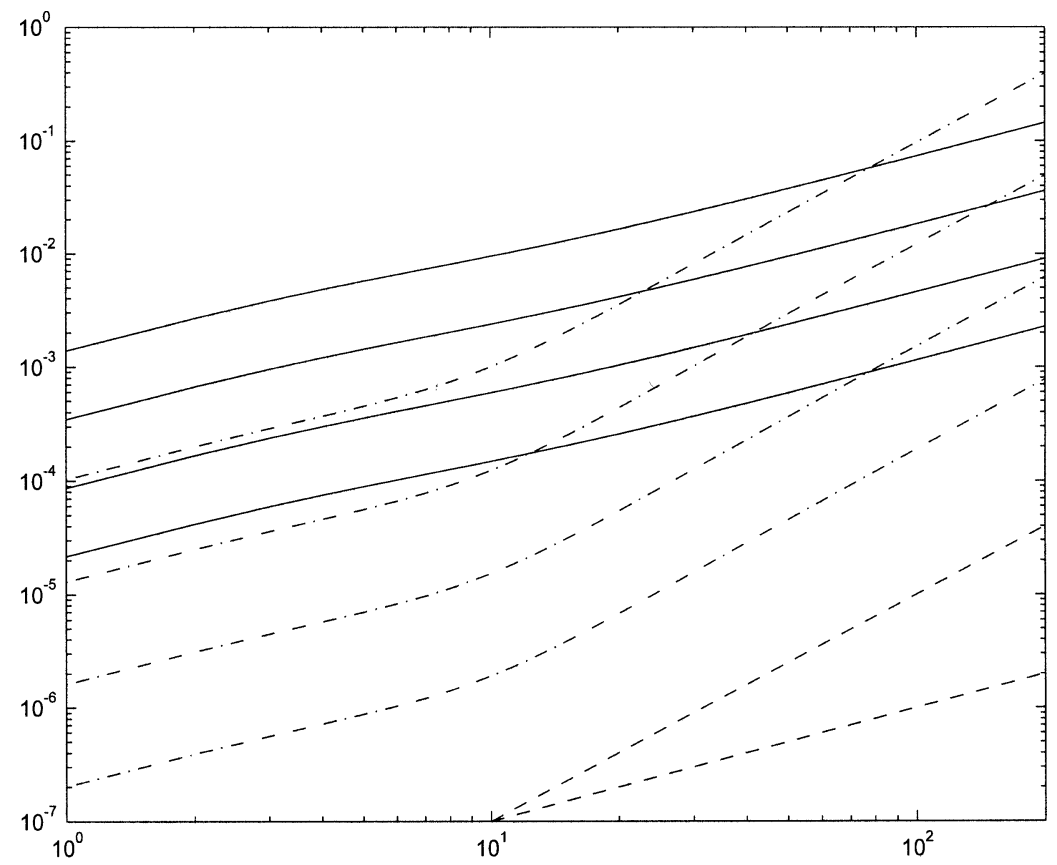

Fig. 2. $L^{2}$-error against $t$. Solid line: $[\mathrm{HCM}]$; dashed line: $[\mathrm{NCM}] . \Delta t=\frac{1}{20}, \frac{1}{40}, \frac{1}{80}, \frac{1}{160}$. The broken lines at the bottom show the slopes for linear and quadratic growth in time. 
Table 1

Errors with respect to the solitary wave at $t=200$

\begin{tabular}{rccc}
\hline \multicolumn{1}{c}{$\Delta t$} & {$[\mathrm{ICM}]$} & {$[\mathrm{HCM}]$} & {$[\mathrm{NCM}]$} \\
\hline $1 \mathrm{E}-01$ & $6.5680 \mathrm{E}-01$ & $5.7426 \mathrm{E}-01$ & - \\
$5 \mathrm{E}-02$ & $1.6527 \mathrm{E}-01$ & $1.4447 \mathrm{E}-01$ & $3.9329 \mathrm{E}-01$ \\
$2.5 \mathrm{E}-02$ & $4.1371 \mathrm{E}-02$ & $3.6164 \mathrm{E}-02$ & $4.9593 \mathrm{E}-02$ \\
$1.25 \mathrm{E}-02$ & $1.0346 \mathrm{E}-02$ & $9.0438 \mathrm{E}-03$ & $6.2069 \mathrm{E}-03$ \\
$6.25 \mathrm{E}-03$ & $2.5866 \mathrm{E}-03$ & $2.2611 \mathrm{E}-03$ & $7.7595 \mathrm{E}-04$ \\
$3.125 \mathrm{E}-03$ & $6.4667 \mathrm{E}-04$ & $5.6530 \mathrm{E}-04$ & $9.6989 \mathrm{E}-05$ \\
\hline
\end{tabular}

Table 2

Errors with respect to the modified solitary wave at $t=200$

\begin{tabular}{rccc}
\hline \multicolumn{1}{c}{$\Delta t$} & {$[\mathrm{ICM}]$} & {$[\mathrm{HCM}]$} & {$[\mathrm{NCM}]$} \\
\hline $1 \mathrm{E}-01$ & $8.1552 \mathrm{E}-02$ & $3.8517 \mathrm{E}-02$ & $1.4539 \mathrm{E}-01$ \\
$5 \mathrm{E}-02$ & $1.9753 \mathrm{E}-02$ & $9.0863 \mathrm{E}-03$ & $3.6307 \mathrm{E}-03$ \\
$2.5 \mathrm{E}-02$ & $4.8981 \mathrm{E}-03$ & $2.2376 \mathrm{E}-03$ & $2.5108 \mathrm{E}-04$ \\
$1.25 \mathrm{E}-02$ & $1.2220 \mathrm{E}-03$ & $5.5732 \mathrm{E}-04$ & $3.1149 \mathrm{E}-05$ \\
$6.25 \mathrm{E}-03$ & $3.0538 \mathrm{E}-04$ & $1.3918 \mathrm{E}-04$ & $3.9005 \mathrm{E}-06$ \\
$3.125 \mathrm{E}-03$ & $7.6328 \mathrm{E}-05$ & $3.4786 \mathrm{E}-05$ & $4.8719 \mathrm{E}-07$ \\
\hline
\end{tabular}

$[\mathrm{ICM}]$ and $[\mathrm{HCM}]$, modified errors behave as $\mathrm{O}\left(\Delta t^{2}\right)$ suggesting that, for the values of $\Delta t$ considered, the complementary term dominates over the remainder; however, the influence of the remainder is noted in the case of [NCM], where modified errors do not have an $\mathrm{O}\left(\Delta t^{3}\right)$ behaviour for moderate values of $\Delta t$ relative to the final time of integration (see the values of the modified error corresponding to [MCN] for $\Delta t=\frac{1}{10}, \frac{1}{20}, \frac{1}{40}$, in Table 2). When we have smaller values of the time step, the complementary term becomes dominant. This seems to show that, for a long final time $t_{\max }$, we need smaller $\Delta t$ to find the leading order of the method in the modified errors.

On the other hand, by making the final time of integration longer and by retaining a fixed $\Delta t$, we have observed that, in the conservative case of [ICM] and [HCM], the bounded behaviour of the complementary term is lost, being substituted by the growth of terms hidden in the remainder. Note that the expansion (32) is not uniform, in the sense that this remainder will in general grow with time [7]. Here we conclude the first type of numerical experiments.

The structure of the numerical solution can be nicely illustrated by plotting the solitary waves. Here, other differences are noted. Fig. 5 shows, at $t=100$, the true solitary wave, with solid line, while the broken line and the crosses represent, respectively, the modified solitary wave and the numerical solution corresponding to $[\mathrm{HCM}]$, with $\Delta t=\frac{1}{20}$. Fig. 6 displays the same elements for [NCM]. In both cases, 


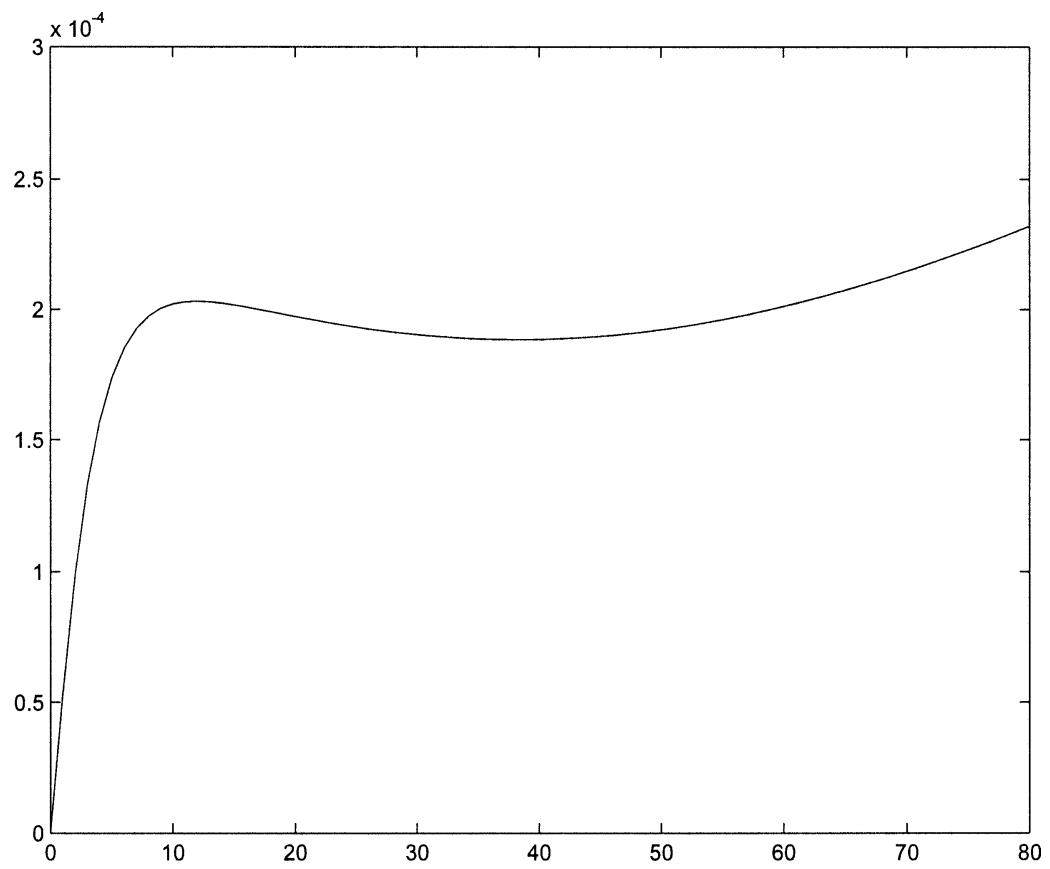

Fig. 3. $L^{2}$-error with respect to the modified solitary wave against $t$. [HCM] with $\Delta t=\frac{1}{80}$.

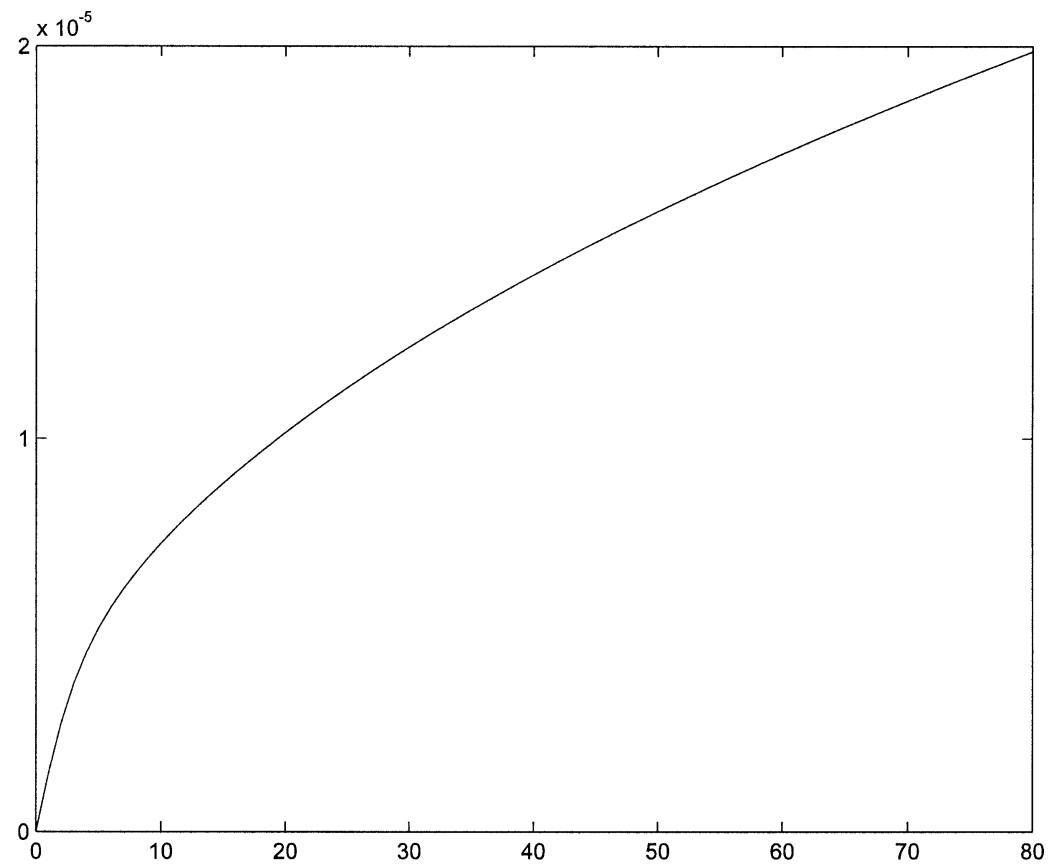

Fig. 4. $L^{2}$-error with respect to the modified solitary wave against $t$. [NCM] with $\Delta t=\frac{1}{80}$. 


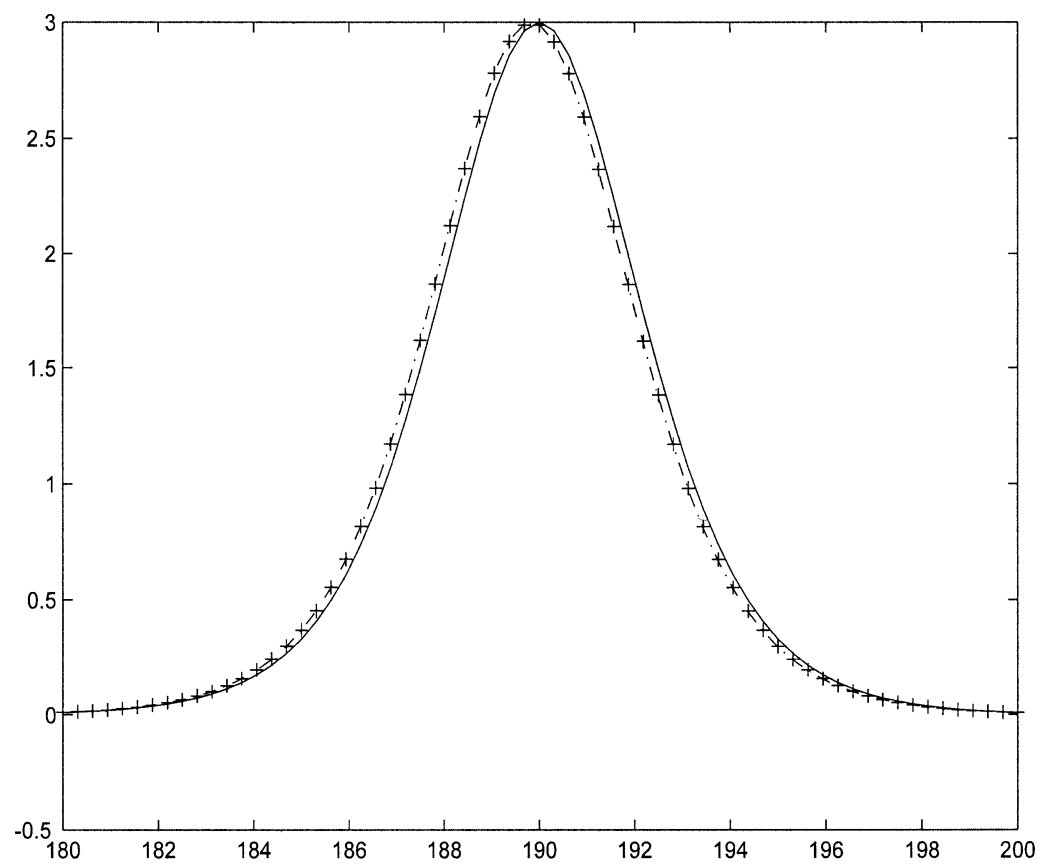

Fig. 5. [HCM] with $\Delta t=\frac{1}{20}$ at $t=100$. Original solitary wave (solid line), modified wave (dashed line) and numerical solution (crosses).

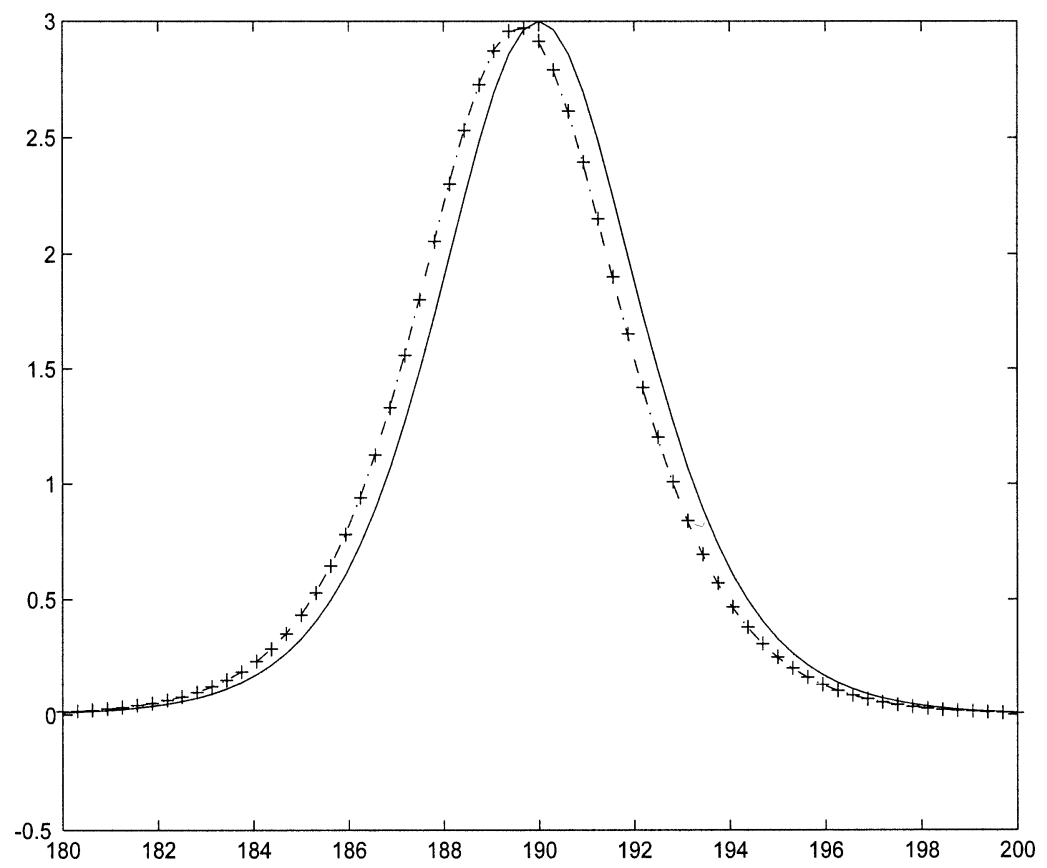

Fig. 6. [NCM] with $\Delta t=\frac{1}{20}$ at $t=100$. Original solitary wave (solid line), modified wave (dashed line) and numerical solution (crosses). 
we see that the numerical solution essentially behaves as a solitary wave, closer to the corresponding modified wave than to the solution (see Tables 1 and 2). For the 'conservative' scheme [HCM], the modified wave differs from the original one in a linear with time perturbation in the initial location (see Theorem 4.1) which in the case of the figure delays its motion with respect to that of the true wave. However, the modified wave keeps the original velocity, and therefore amplitude. In fact, the modified wave associated to $[\mathrm{HCM}]$ also preserves the value of the 'mass' of the original solitary wave, represented by the Casimir function $C$,

$$
C(\psi)=12 \sqrt{c(c-1)}
$$

because this quantity only depends on the velocity $c$.

This is not the situation in the case of the 'nonconservative' method [NCM]. Here, the modified wave has a different velocity of propagation and, therefore, amplitude, see (33), growing linearly with time with respect to the velocity of the solution. This is visible in Fig. 6. In particular, the modified wave does not conserve the 'mass' $C$. Since the numerical solution does preserve it, the mass lost in the approximation to the modified solitary wave is recovered in the formation of a tail [9] behind the numerical solitary wave profile. This tail can be observed in Fig. 7, which displays the numerical solution appearing in Fig. 6, but with a vertical scale with some more degrees of magnitude. This phenomenon does not occur in the conservative case.

Finally, we complete the numerical experiments by analyzing the behaviour, through the numerical integration, of the conserved quantities $I$ and $H$. We study the evolution of the differences

$$
\begin{aligned}
& H\left(U^{n}\right)-H\left(\psi\left(t_{n}\right)\right)=H\left(U^{n}\right)-H\left(U^{0}\right), \\
& I\left(U^{n}\right)-I\left(\psi\left(t_{n}\right)\right)=I\left(U^{n}\right)-I\left(U^{0}\right),
\end{aligned}
$$

between the values of $I$ and $H$ at the solitary wave solution and the discrete versions of the quantities, at the numerical solution given by $[\mathrm{HCM}]$, for (38), and by [NCM], for both (37) and (38). These discrete versions of the invariants use the pseudospectral spatial discretization and are good approximations of the quantities at the corresponding semidiscrete numerical solution [25]. Observe that, since $I$ and $H$ are conserved quantities of the problem, the differences (37) and (38) also estimate the evolution of $I$ and $H$ in the numerical integration. Figs. 8 and 9 represent, respectively, the evolution of $I$ and $H$ at the numerical solution given by [NCM], while Fig. 10 shows the values (38) in the case of [HCM].

Recall that, in general, the difference between an invariant of the problem considered at the numerical approximation and at the true solution is of the order of the method [9]; if we expand this difference in powers of $\Delta t$, the coefficient of the leading term is the inner product between the gradient of the invariant at the true solution and the leading term of the asymptotic expansion of the global error. This dominant order can be seen, in the case of [NCM], in Figs. 8 and 9, where errors (37) and (38) show an $\mathrm{O}\left(\Delta t^{3}\right)$ behaviour. However, as far as [HCM] is concerned, note that, the leading term of its local error is orthogonal not only to the gradient of $H$ at $\psi$ (because the scheme is Hamiltonian conserving) but also to the gradient of the other quantity $I$ at the solitary wave (because of the relative equilibrium condition (9)). This fact and the conservation of $I$ by the solution $\psi$ implies $[9,12]$ that the leading term of the global error is also orthogonal to that gradient; then as $\Delta t \rightarrow 0$ with $t_{n}$ fixed, the order of the error (38) is $\mathrm{o}\left(\Delta t^{2}\right)$. Fig. 10 shows an $\mathrm{O}\left(\Delta t^{4}\right)$ behaviour. 


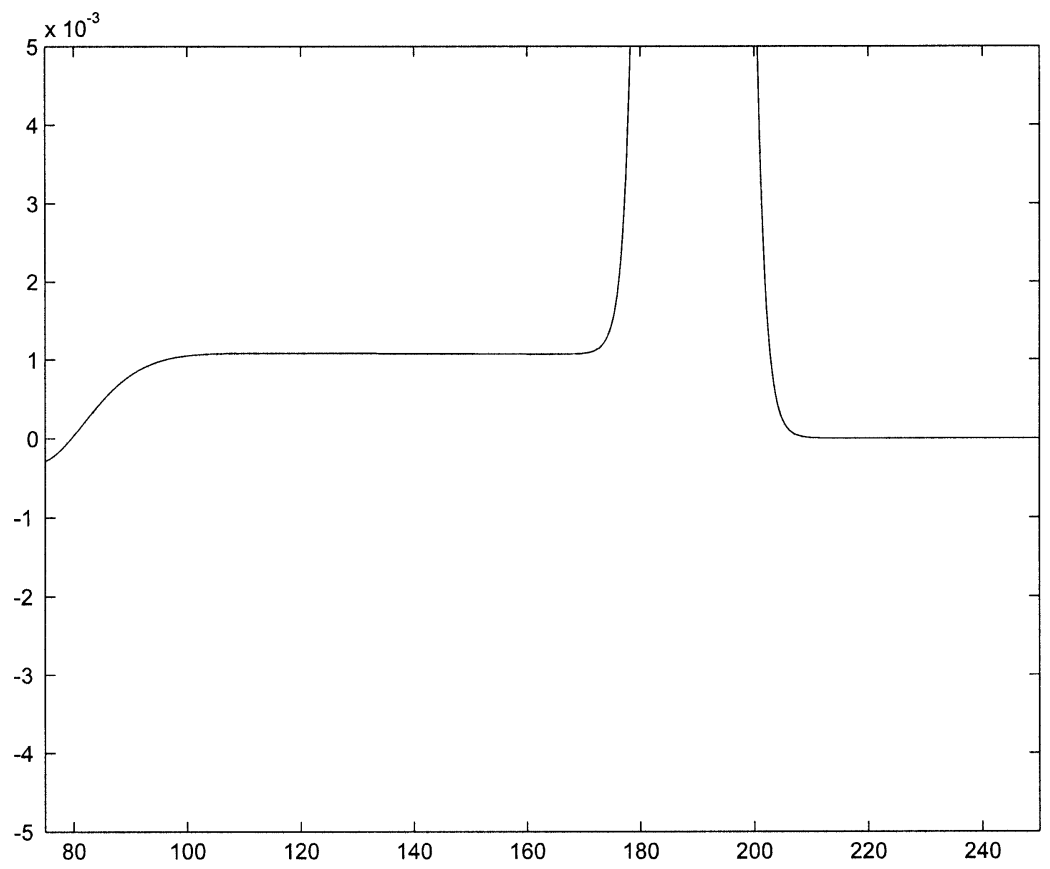

Fig. 7. [NCM] with $\Delta t=\frac{1}{20}$ at $t=100$. Numerical solution with magnified vertical scale. A tail is formed.

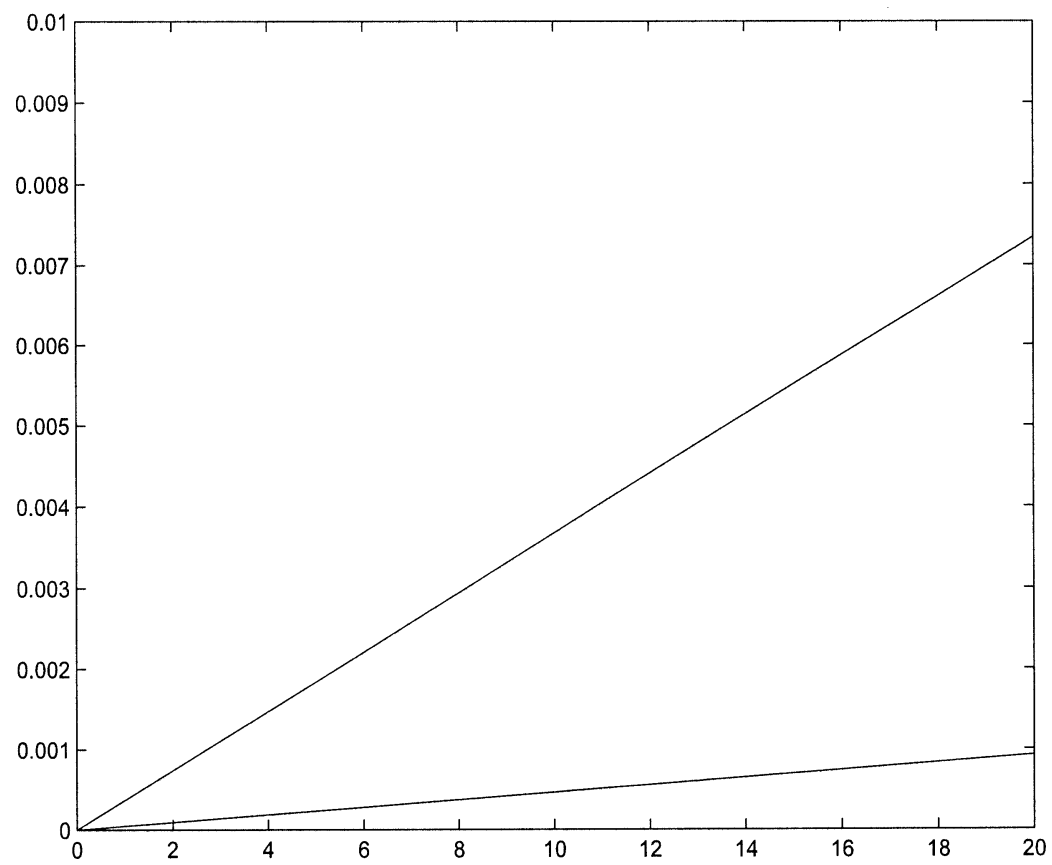

Fig. 8. Momentum error against $t$. [NCM] with $\Delta t=\frac{1}{20}, \frac{1}{40}$. 


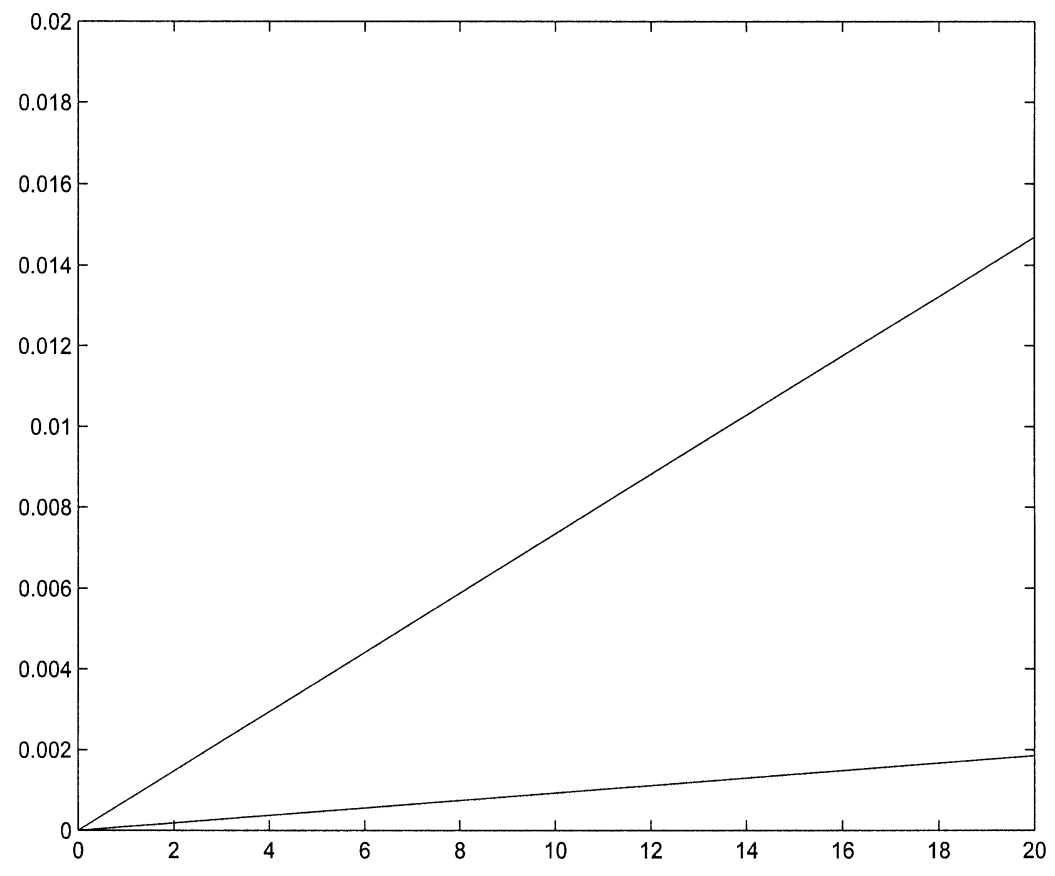

Fig. 9. Energy error against $t$. [NCM] with $\Delta t=\frac{1}{20}, \frac{1}{40}$.

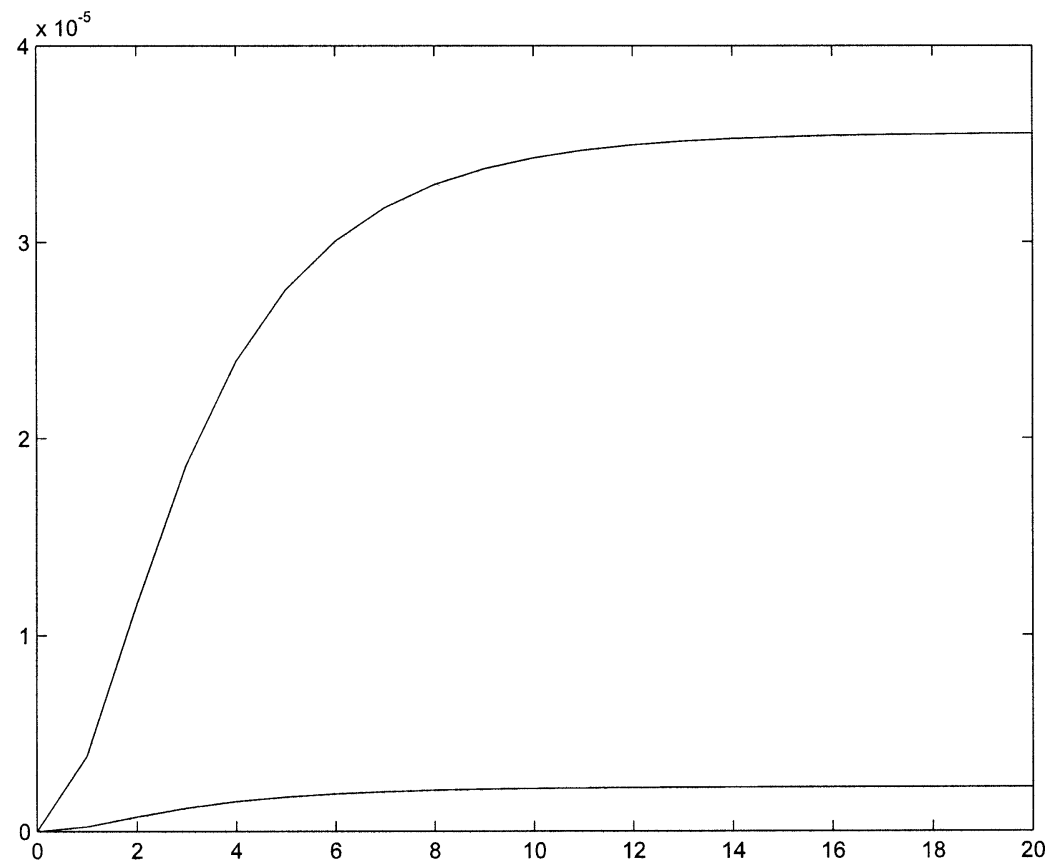

Fig. 10. Momentum error against $t$. [HCM] with $\Delta t=\frac{1}{10}, \frac{1}{20}$. 


\section{Acknowledgements}

This research has been supported by the Center of Mathematics, Universidade de Coimbra. A.D. is very grateful for the hospitality of the Department of Mathematics of this university, where this work was done.

\section{References}

[1] V.I. Arnold, Mathematical Methods of Classical Mechanics, Springer, Berlin, 1989.

[2] T.B. Benjamin, The stability of solitary waves, Proc. R. Soc. London Ser. A 328 (1972) 153-183.

[3] T.B. Benjamin, J.L. Bona, J.J. Mahoney, Model equations for long waves in nonlinear dispersive systems, Philos. Trans. R. Soc. London Ser. A 272 (1972) 47-78.

[4] J.L. Bona, A. Soyeur, On the stability of solitary wave solutions of model equations for long waves, J. Nonlinear Sci. 4 (1994) 449-470.

[5] M.P. Calvo, E. Hairer, Accurate long-term integration of dynamical systems, Appl. Numer. Math. 18 (1995) 95-105.

[6] M.P. Calvo, J.M. Sanz-Serna, The development of variable-step symplectic integrators with application to the two-body problem, SIAM J. Sci. Comput. 14 (1993) 936-952.

[7] B. Cano, J.M. Sanz-Serna, Error growth in the numerical integration of periodic orbits, with application to Hamiltonian and reversible systems, SIAM J. Numer. Anal. 34 (1997) 1391-1417.

[8] W. Craig, M. Groves, Hamiltonian long-wave approximations to the water-wave problem, Wave Motion 19 (1994) 367-389.

[9] J. de Frutos, J.M. Sanz-Serna, Accuracy and conservation properties in numerical integration: the case of the Korteweg-de Vries equation, Numer. Math. 75 (1997) 421-445.

[10] P.G. Drazin, R.S. Johnson, Solitons. An Introduction, Cambridge Texts in Applied Mathematics, Cambridge University Press, Cambridge, 1990.

[11] A. Durán, J.M. Sanz-Serna, The numerical integration of relative equilibrium solutions. Geometric theory, Nonlinearity 11 (1998) 1547-1567.

[12] A. Durán, J.M. Sanz-Serna, The numerical integration of relative equilibrium solutions. The nonlinear Schrödinger equation, to appear in IMA J. Numer. Anal.

[13] J.C. Eilbeck, G.R. McGuire, Numerical study of the regularized long-wave equation II: interaction of solitary waves, J. Comput. Phys. 23 (1977) 63-73.

[14] D.J. Estep, A.M. Stuart, The rate of error growth in Hamiltonian conserving integrators, Z. Angew. Math. Phys. 46 (1995) 407-418.

[15] L.R.T. Gardner, G.A. Gardner, Solitary waves of the regularised longwave equation, J. Comput. Phys. 91 (1990) 441-459.

[16] L.R.T. Gardner, G.A. Gardner, Solitary waves of the equal width wave equation, J. Comput. Phys. 101 (1992) 218-223.

[17] C.W. Gear, Invariants and numerical methods for ODEs, Phys. D 60 (1990) 303-310.

[18] E. Hairer, S.P. Nørsett, G. Wanner, Solving Ordinary Differential Equations I, Nonstiff Problems, 2nd Edition, Springer, Berlin, 1993.

[19] T. Itoh, K. Abe, Hamiltonian-conserving discrete canonical equations based on variational difference quotients, J. Comput. Phys. 77 (1988) 85-102.

[20] J.E. Marsden, T.S. Ratiu, Introduction to Mechanics and Symmetry, Springer, New York, 1994.

[21] J.R. Miller, M.I. Weinstein, Asymptotic stability of solitary waves for the regularized long-wave equation, Comm. Pure Appl. Math. 49 (1996) 399-441. 
[22] P.J. Morrison, J.D. Meiss, J.R. Cary, Scattering of regularized-long-wave solitary waves, Phys. D 11 (1984) 324-336.

[23] P.J. Olver, Applications of Lie Groups to Differential Equations, 2nd Edition, Springer, New York, 1993.

[24] P.J. Olver, Euler operators and conservation laws of the BBM equation, Math. Proc. Cambridge Philos. Soc. 85 (1979) 143-160.

[25] D. Pathria, J.L. Morris, Pseudospectral solution of nonlinear Schrödinger equations, J. Comput. Phys. 87 (1990) 108-125.

[26] R. Pego, M.I. Weinstein, Asymptotic stability of solitary waves, Comm. Math. Phys. 164 (1994) 305-349.

[27] D.H. Peregrine, Calculations of the development of an undular bore, J. Fluid Mech. 25 (1996) 321-330.

[28] J.M. Sanz-Serna, Geometric integration, in: I.S. Duff, G.A. Watson (Eds.), The State of the Art in Numerical Analysis, Clarendon Press, Oxford, 1997, pp. 121-143.

[29] J.M. Sanz-Serna, M.P. Calvo, Numerical Hamiltonian Problems, Chapman-Hall, London, 1994.

[30] D.M. Sloan, Fourier pseudospectral solution of the regularised long wave equation, J. Comput. Appl. Math. 36 (1991) 159-179.

[31] D. Stoffer, Variable steps for reversible integration methods, Computing 55 (1995) 1-22.

[32] W.A. Strauss, 1. Vázquez, Numerical solution of a nonlinear Klein-Gordon equation, J. Comput. Phys. 28 (1978) 271-278.

[33] M.I. Weinstein, Modulational stability of ground states of nonlinear Schrödinger equations, SIAM J. Math. Anal. 16 (1985) 473-491.

[34] M.I. Weinstein, Existence and dynamic stability of solitary wave solutions of equations arising in long wave propagation, Comm. Partial Differential Equations 12 (1987) 1133-1175.

[35] G.B. Whitham, Linear and Nonlinear Waves, Wiley, New York, 1974. 